\title{
Transnacionalización de la educación docente: ¿un nuevo paradigma para Ontario?
}

\section{Transnationalization of Teacher Education: A New Paradigm for Ontario?}

\author{
LaTransnationalisation de l'éducation \\ des enseignants : \\ Un nouveau paradigme pour l'Ontario?
}

\section{LeRoy Whitehead}

Queen's University, Canadá

\section{RESUMEN}

El propósito de este artículo es hacer una evaluación informal del grado en que los conceptos de la transnacionalización son viables en programas de formación inicial de docentes en la provincia de Ontario, Canadá, utilizando el programa de una Facultad de Ontario como caso de prueba. Analizo dos artículos seleccionados con el propósito de identificar algunos de los temas o de las características más destacadas de los programas transnacionalizados de formación del profesorado y de docentes efectivos que trabajan en entornos transnacionalizados. El programa que sirve como caso de prueba se analiza para determinar cuáles de estos temas o características están presentes en él, o si en definitiva los hay. Identifico los factores que bien pueden promover u obstaculizar los pasos hacia la transnacionalización del programa. La conclusión es que la transnacionalización puede ser un paradigma viable para la formación de docentes en Ontario.

Descriptores: transnacionalización; educación docente (formación inicial); Ontario; facultad de educación.

\section{ABSTRACT}

The purpose of this article is to make an informal assessment of the degree to which concepts of transnationalization are viable in pre-service teacher education programs in the Province of Ontario, Canada, using one Ontario Faculty's program as a test case. Two selected articles are analyzed for the purpose of identifying some salient themes or characteristics of transnationalized teacher education programs and of effective teachers working in transnationalized settings. The test case program is then analyzed to identify which, if any, of these themes or characteristics are present in the program. Factors that may either promote or impede further steps toward 
transnationalization of the program are identified. The conclusion is that transnationalization could be a viable paradigm for teacher education in Ontario.

Key words: transnationalization; teacher education (pre-service teacher education); Ontario; faculty of education.

\section{RÉSUMÉ}

Le but de cet article est de faire une évaluation informelle pour déterminer jusqu'à quel point les concepts de transnationalisation sont viables dans les programmes d'éducation de base (préservice) des enseignants dans la province de l'Ontario, au Canada, en utilisant un des programmes de la Faculté de L'Ontario comme cas d'essai. Deux articles sont choisis et analysés dans le but d'identifier des thèmes saillants ou des caractéristiques de programmes d'éducation transnationalisés, et des enseignants efficaces œuvrant dans des cadres transnationalisés. Le programme des cas d'essai est ensuite analysé pour identifier lesquels de ces thèmes ou caractéristiques, s'il y en a, sont contenus dans le programme officiel de base. Les facteurs qui pourraient promouvoir ou empêcher le progrès vers la transnationalisation du programme sont identifiés. La conclusion est que la transnationalisation pourrait être un paradigme viable pour l'éducation des enseignants en Ontario.

Mots clés : La transnationalisation; L'éducation des enseignants (préservice); L'Ontario; la faculté d'éducation.

$\mathrm{C}_{\mathrm{r}}^{\circ}$ ON EL FIN DE SER TOTALMENTE hONESTO, debería advertir a los lectores que no pretendo ser un "experto", ya sea en el ámbito de la transnacionalización o en la teoría de la formación de docentes. Lo que sí puedo ofrecer es una experiencia muy práctica obtenida durante una carrera de cincuenta años hasta la fecha en la enseñanza en escuelas públicas y en la formación inicial de maestros y profesores de media. En particular, tuve el honor de servir como decano asociado de mi Facultad de Educación entre 1994 y 2008. ${ }^{1}$ Durante ese tiempo, estuve a cargo de administrar las operaciones cotidianas del programa de formación docente de pre-grado de la facultad de educación. Luego de dejar el cargo, he seguido enseñando en el programa. En lugar de un artículo "académico" tradicional, presentaré una reflexión informal y personal, destilada a partir de mi experiencia, mis lecturas y el pensamiento acerca de las escuelas, la educación y los programas de formación inicial docente. ${ }^{2}$ Espero que esta contribución pueda agregar una dimensión adicional al esfuerzo colectivo y tal vez ofrecer algunas perspectivas útiles o formas de pensar sobre el tema.

Mi propósito al escribir este artículo es hacer una evaluación informal de la medida en que los conceptos de la transnacionalización son viables en programas de formación inicial de docentes en la provincia de Ontario, Canadá. Utilizaré el programa de formación docente de mi propia facultad, aquella con la que estoy más familiarizado, como caso de estudio. En primer lugar, repasaré brevemente dos artículos que he encontrado útiles para el propósito de identificar algunos de los temas más destacados o las características presentes en programas de formación docente transnacionalizados y en docentes efectivos que trabajan en entornos transnacionalizados. En segundo lugar, identificaré, si es que los hay, cuáles de estos temas o características están presentes en nuestro programa. En tercer lugar, definiré los factores que pueden favorecer u obstaculizar los pasos posteriores hacia la transnacionalización del programa y, finalmente, presentaré algunas conclusiones. 


\section{Transnacionalización del currículum en derecho: Cómo enseñar lo que vivimos (Jukier, 2006)}

La literatura de la transnacionalización profundiza en la discusión del transnacionalismo como un fenómeno socio-político y en la discusión acerca de cómo este fenómeno ha afectado y seguirá afectando a los sistemas escolares, sin embargo, es relativamente superficial en términos de consejos prácticos o perspectivas sobre la forma que debería tener un paradigma transnacionalista para la formación docente, o el tipo de proceso que se podría poner en marcha para transformar un programa convencional de formación docente en uno de características más transnacionales. Curiosamente, uno de los artículos más perspicaces y útiles que he encontrado en este último sentido no trata sobre la formación docente, sino que sobre la educación legal. Jukier (2006) presenta un estudio de caso elocuente en el que describe la transformación de los programas de educación legal de una facultad de derecho de Canadá de un régimen convencional a uno transnacional (o, como lo prefiere la facultad, "transistémico").

La formación del profesorado y la formación jurídica pueden ser diferentes en muchos aspectos. Sin embargo, incluso teniendo en cuenta esas diferencias, la documentación de Jukier sobre la transformación del programa en la Universidad McGill de Montreal, Canadá, merece una consideración cuidadosa por parte de los formadores de docentes, porque incluye varias ideas importantes y principios rectores que podemos recoger. No entregaré un resumen detallado del artículo aquí ya que es de fácil acceso en línea e insto a la lectura del mismo. También deben saber esto: la transformación del programa en McGill fue real y radical, no se quedó sólo en las palabras o en el re envasado de cursos antiguos con etiquetas nuevas, ni tampoco fue un cambio de marca superficial con cursilerías y líneas publicitarias inteligentes en anuncios para atraer a los estudiantes que, de lo contrario, podrían ir a otra parte. ${ }^{3}$ En mi opinión, lo primero y quizás lo más importante que la comunidad educadora de docentes puede aprender de la experiencia de McGill es lo siguiente: si queremos ofrecer programas realmente transnacionalizados (o transistémicos) para la formación del profesorado, debemos estar preparados para llevar a cabo una transformación genuina de estos. Meramente re envasar o re estructurar antiguos memes no será suficiente.

Un elemento crucial en el proyecto de McGill ofrece el segundo principio fundamental para los formadores de docentes, ya que conduce al corazón del concepto de formación profesional transistémica: la facultad de McGill, trató de "incorporar el transnacionalismo en el currículo mediante la liberación de los estudios de derecho de las barreras jurisdiccionales o sistémicas"(p. 174, énfasis añadido). Esta idea causará gran revuelo, ya sea positiva o negativamente, entre los formadores de docentes quienes están muy acostumbrados a adaptar sus programas de formación docente a las especificaciones establecidas por sus respectivos ministerios de educación. Jukier continúa: “... la ambición es crear juristas cosmopolitas. El plan de estudios está diseñado para ampliar las perspectivas sobre la educación jurídica con el fin de desarrollar mentes más ágiles en nuestros estudiantes, al igual que abogados y profesionales del 
derecho más enfocados hacia el exterior y, capacitados de manera más integral entre nuestros egresados. "(P. 174, énfasis añadido)

¿Pueden los formadores de docentes verdaderamente aspirar a liberar a la educación de docentes de los límites jurisdiccionales o sistémicos y crear educadores cosmopolitas con perspectivas profesionales amplias y mentes ágiles, más orientados hacia el exterior y entrenados de manera integral? Podríamos percibir que la formación de docentes está más íntimamente relacionada con los gobiernos jurisdiccionales que con la educación jurídica, pero McGill también debió asegurarse de que su programa de educación legal cumplía con los requerimientos jurisdiccionales y las expectativas de los futuros empleadores acerca de los graduados. La forma en que la transformación de McGill cumple y supera las regulaciones y las expectativas puede ser de utilidad para los formadores de docentes.

De manera histórica, la Universidad McGill ha ofrecido dos programas diferentes en educación legal: uno para el enfoque legal británico común y otro enfocado en el código civil francés. Ambos sistemas legales presentan diferentes estructuras de pensamiento, se basan en tradiciones intelectuales distintas y los valores y principios trascendentes que reflejan también difieren. El programa modificado combina el estudio de estos sistemas divergentes en un programa único e integral que otorga a los graduados dos grados académicos de licenciatura en el área legal: uno en derecho civil (BCL o Bachelor in Civil Law) y uno en derecho general (LLB, o Bachelor of Laws); cada uno de estos programas se ajusta al marco regulatorio, sin embargo debido a la manera en que el estudio de ambos sistemas legales se integra, mediante un enfoque comparativo, el nuevo programa va más allá de dichas regulaciones y prepara profesionales del derecho mucho más cosmopolitas. Se sobre entiende que el enfoque comparativo que se adoptó no es ni simplista ni superficial. Dado que ni las tradiciones filosóficas subyacentes, ni los conceptos básicos, ni los detalles de ambos sistemas legales se corresponden de ninguna manera conveniente, no se puede establecer comparaciones superficiales entre ellos. La integración demandó toda una re conceptualización y un rediseño de los cursos lo que requirió un trabajo conjunto de profesores de derecho civil y de derecho constitucional y, en su etapa inicial, una enseñanza conjunta, al igual que el desarrollo de nuevos materiales para la enseñanza y el aprendizaje. Las comparaciones complejas entre diferentes tradiciones y filosofías legales, además de la deducción de conexiones entre estas filosofías y las leyes que producen como resultado generan una comprensión mucho más amplia y profunda de la ley de los conceptos legales que la que se podría obtener en otro escenario.

Jukier estima que el aspecto comparativo de la educación legal transistémica es vital. Lo que hace que el enfoque transistémico de la ley comparada sea distinto de otros enfoques convencionales de leyes comparadas es la aproximación integrada, más que secuencial, a ambas tradiciones y el énfasis en establecer relaciones entre las tradiciones legales y sus perspectivas resultantes. (p. 176) La autora indica:

Lo que se necesita es un programa de estudios que pueda estimular las mentes para que se vuelvan tan ágiles y creativas que puedan pensar con apertura dentro de marcos alternos de pensamiento, moviéndose con destreza entre ellos y 
traspasando sus barreras, como debe ser... Al trabajar con diferentes tradiciones legales, cada una con diversos fundamentos históricos y metodológicos, los estudiantes son expuestos a una variedad de perspectivas y análisis contextuales de problemas legales. El objetivo es perfeccionar las habilidades de los estudiantes en cuanto a la comprensión imaginativa, a la vez que se debilita la noción de que hay una estructura única de la realidad. (p. 177)

Los estudiantes utilizan materiales legislativos, jurisprudencia y doctrina de diversos países, sin embargo este enfoque transistémico es mucho más que simple ley comparada. Debe ir "más allá de los confines de formas convencionales y relacionarse con una búsqueda filosófica en la justicia de las relaciones que se expresan dentro de estas formas legales." (p. 178, citando a Janda, 2005)

De esta manera, el tercer y cuarto principios guía parecen ser la integración tanto del estudio de enfoques comparativos (o alternos) para la enseñanza y el aprendizaje, como de las tradiciones filosóficas de las cuales surgen estos enfoques. El quinto sería la incorporación de materiales de aprendizaje de otros países. (p. 180)

El sexto elemento que podemos rescatar de la experiencia McGill es que la transnacionalización de la educación de docentes tomará tiempo, probablemente años; será un proceso, no un evento. Al momento en que Jukier escribió su artículo, el proyecto McGill estaba en su séptimo año de ejecución, aunque la autora resume una amplia pre historia: "Es complicado localizar con precisión un momento específico en que este concienzudo proceso de auto reflexión se inició," indica, "pero en 1994, un currículum integrado y comparativo fue tema de discusión en una jornada de retiro fuera de la facultad." (p. 182) Cuatro años y medio de comités ad-hoc, discusiones y debates tomaron lugar hasta que se aprobaron los cambios propuestos a nivel de facultad y dirección de la universidad. El currículum integrado y comparativo resultó ser la característica central del nuevo programa de McGill.

El séptimo elemento que identificamos es que se necesitará un liderazgo fuerte e incansable. En el caso de McGill, el liderazgo del decano de la facultad mantuvo el proyecto en movimiento. (p. 183)

El octavo punto también surge de la pre historia del programa de McGill, al igual que de las circunstancias únicas de la universidad. El programa de educación en derecho exclusivo de McGill surgió en parte del hecho que Montreal se ubica en la provincia de Quebec. Por motivos históricos y culturales, mucho del sistema legal de Quebec se basa en conceptos del derecho civil arraigados en el código civil francés, el cual es muy diferente del enfoque cercano al derecho consuetudinario inglés presente en el resto del país. El punto es que el programa se desarrolló como resultado de factores únicos, como la ubicación de la universidad, la situación socio-política, además del conjunto de programas profesionales y el perfil experto de los miembros de la facultad de derecho de McGill. Es probable que nuestras facultades de educación no compartan el mismo contexto histórico, circunstancial o de experticia; sin embargo, tenemos historias, circunstancias y perfiles de experticia propios y únicos que podemos utilizar como punto de inicio, además de recursos para crear programas exclusivos y transnacionalizados de educación docente. ¿Quién dice que sólo hay una 
manera o incluso "la mejor manera" de transnacionalizar la educación docente?

Jukier resume siete desafíos presentes en este tipo de transformación curricular de la siguiente manera:

- Idioma. Es de utilidad si tanto los profesores como los estudiantes pueden leer en más de un idioma para aumentar el acceso a material legal extranjero.

- Experticia de la facultad. Puede ser necesario que los miembros de la facultad desarrollen experticia adicional con el fin de implementar un programa de este tipo.

- Materiales de enseñanza. Cuando se lanzó el programa, había una escasez de materiales de aprendizaje diseñados de manera adecuada al programa.

- Organización del curso. Las propuestas de cursos existentes tienden a basarse en el enfoque ortodoxo. Puede ser necesario rediseñar completamente los cursos, cuando no completamente re conceptualizarlos.

- Evaluación del rendimiento de los estudiantes. Para proponer cursos nuevos e innovadores puede ser necesario desarrollar formas nuevas e innovadoras de evaluar el aprendizaje de los estudiantes.

- Aceptación interna y externa. Una transformación de gran envergadura requiere mucha discusión y planificación académica. Todos los grupos involucrados, como estudiantes, ex alumnos y potenciales empleadores de los graduados deben estar informados y educados acerca de los cambios en el programa.

- Desafíos financieros. Se debe tomar una decisión consciente de destinar recursos a las nuevas prioridades. (ver pp. 180-182)

\section{Características de los profesores para la educación efectiva en las tierras fronterizas (Cline y Necochea, 2006)}

El estudio de caso desarrollado por Jukier nos entrega algunas perspectivas importantes acerca de posibles características relevantes que debería presentar un programa de educación docente transnacionalizado. Otro artículo de características bastante diferentes nos entrega perspectivas en cuanto a las disposiciones o características que los profesores de las "tierras fronterizas" necesitan para ser eficientes en su trabajo con niños y grupos familiares transnacionalizados. (Cline y Necochea, 2006) En el caso del artículo de Cline y Necochea, el concepto de "zonas fronterizas" se refiere al área que se encuentra a ambos lados de la frontera entre México y Estados Unidos, pero particularmente a aquellas áreas de los Estados Unidos en que un gran porcentaje de la población es de origen latino o hispano. ${ }^{4}$ Aunque el artículo presenta los resultados de un estudio prospectivo, sus resultados merecen consideración y parecen estar alineados con los hallazgos de otros investigadores. Nuevamente, no entregaré un resumen detallado del informe acá, ya que es fácil acceder a él en línea y recomiendo 
su lectura. Si consideramos que las disposiciones personales identificadas en el estudio son válidas, entonces el proceso de admisión en los programas de educación de docentes debería buscar la admisión de los candidatos que ya poseen estas disposiciones hasta cierto punto, o bien deberíamos tratar de desarrollarlas durante el programa, o ambas.

El estudio consideró a 20 profesores de San Diego y 20 de Tijuana quienes asistieron a 10 sesiones mensuales del Border Pedagogy Biliteracy Institute entre 2003 y 2004. Los datos disponibles como resultado de las reflexiones, evaluaciones, retroalimentación y materiales de instrucción que produjeron los participantes se recopilaron de manera permanente durante las sesiones de capacitación. El estudio utilizó la teoría fundamentada y los procedimientos analíticos. A partir de los datos, se identificó cinco características necesarias para enseñar en las tierras fronterizas; presentaré estas cinco características acompañadas de una breve cita de cada una para ilustrar la naturaleza de los hallazgos.

- Apertura de mente y flexibilidad. "Las escuelas de zonas fronterizas con frecuencia deben enfrentarse a lo inesperado, como por ejemplo la repentina llegada de Mixtecos desde Oaxaca. De esta manera, los profesores deben ser abiertos de mente y flexibles en cuanto a los estudiantes bajo su tutela y al currículum que diseñan e implementan. Las estructuras y prácticas instruccionales tradicionales a menudo no son efectivas para alumnos que viven en territorios fronterizos... así, los profesores deben plantearse una escolaridad más efectiva, en ocasiones sin un descriptor o bosquejo a seguir." (p. 272)

- Pasión por educar en contexto fronterizo. "Los profesores efectivos de las zonas fronterizas son apasionados acerca de su labor y hacen lo que sea necesario para ayudar a los estudiantes transnacionales a alcanzar el éxito. Tienen una actitud positiva hacia el trabajo con comunidades fronterizas y buscan prácticas instruccionales que sean efectivas para sus estudiantes... ellos suplen las necesidades de los estudiantes al comprender e interiorizarse de la realidad de las 'familias y las comunidades' de los estudiantes." (pp. 273-274)

- Desarrollo profesional continuo. El desarrollo profesional que se enfoca en la comprensión de las influencias transnacionales que impactan a los estudiantes, las familias y las comunidades es particularmente importante porque entrega a los educadores de zonas fronterizas el conocimiento y las capacidades para diseñar prácticas instruccionales efectivas.” (p. 276)

- Sensibilidad cultural. "La sensibilidad cultural expresada por los participantes del estudio fue más allá de una base superficial de conocimientos acerca de diversas culturas. Los participantes discutieron una comprensión profunda de la cultura que incluía no sólo conocer a las familias, a las comunidades y a los estudiantes de sus cursos, sino que también aceptar y reforzar su cultura y sus maneras de descubrir y comprender el mundo. Los participantes valoraban a aquellos que son diferentes y trabajaban por una mayor unidad de culturas, clases y grupos socioeconómicos." (p. 276) 
- Orientación lingüistica pluralista. Los profesores efectivos fomentan la incorporación de diversas lenguas primarias en el aula e implementan estrategias instruccionales innovadoras para honrar y valorar la lengua nativa de cada estudiante ... con frecuencia utilizan el apoyo de la lengua primaria para asegurarse de que los estudiantes accedan a los conceptos fundamentales del currículum y sean capaces de dominar conceptos complejos ... no consideran el lenguaje como una desventaja o algo que deba ser fijo, sino que como un increíble recurso que se debe trabajar y fomentar, incluso hasta el punto de aprender ellos mismo la lengua. (pp. 278-279)

\section{Temáticas comunes}

El nivel de coincidencias en los temas surgidos en los dos artículos revisados es sorprendente:

- La necesidad de superar las fronteras, sean estas sistémicas y jurisdiccionales o bien se trate de formas y estructuras curriculares y pedagógicas;

- La necesidad de tener una mirada orientada a lo externo, abiertos de mente, con mayor conciencia acerca de las problemáticas relacionadas con la diversidad cultural y con mayor aceptación de otras culturas, además de la capacidad de pensar de manera alternada en sistemas de valores y cultura diversos sin caer en los prejuicios;

- adoptar un enfoque comparativo, aprovechando las diferencias culturales y lingüísticas que pudiese existir en la sala para mejorar el aprendizaje en vez de enfrentarlas como limitaciones;

- la necesidad de un conocimiento profundo, más que superficial, de las culturas, las tradiciones y los valores de los estudiantes;

- la utilidad del hecho que el profesor comprenda más de un idioma y que valore el multilingüismo en el aula;

- el énfasis en el desarrollo profesional constante, la práctica reflexiva y el desarrollo de comunidades de aprendizaje; y

- fuertes contracorrientes, orientadas a la justicia social.

\section{Patrones de la formación docente en Ontario}

Puede ser de utilidad para el lector si explico algunas cosas antes de continuar.

En la Provincia de Ontario, el programa básico de educación inicial docente consta de ocho meses, luego de completar un programa de pregrado. Generalmente, este programa pregrado se trata de una Licenciatura de cuatro años (es decir, ocho semestres), aunque también otros grados son aceptables; por otra parte, el programa 
de ocho meses es equivalente a dos semestres y se desarrolla habitualmente entre septiembre y abril. ${ }^{5}$ En el caso de nuestra Facultad de Educación, casi la mitad de este tiempo, o el equivalente a un semestre, está dedicado a cursos basados en el campus, mientras que la otra mitad, equivalente a otro semestre, está dedicada a experiencias prácticas que se llevan a cabo en tres bloques durante el período de los ocho meses: dos bloques durante el primer semestre (de septiembre a diciembre) y un bloque más largo durante el segundo semestre (enero a abril). Los dos bloques del primer semestre y parte del bloque del segundo semestre están dedicados a desarrollar experiencias prácticas en escuelas primarias y secundarias asignadas por la Facultad. La parte restante del bloque del segundo semestre se dedica a una pasantía de "práctica alterna" en una institución elegida por el candidato a docente y aprobada por la Facultad. Para esta práctica, el candidato debe seleccionar una experiencia que entregue una perspectiva acerca de la educación que sea distinta a aquella que enfrenta un profesor de aula en Ontario. Este instructivo es vago con la intención de proveer un amplio abanico de posibilidades y muchos de nuestros candidatos han sido muy creativos.

Mientras escribo este estudio, el gobierno de Ontario se encuentra discutiendo con varios grupos de interés, incluyendo a las facultades de educación, en cuanto a la extensión del programa de formación inicial previo al ejercicio profesional y con miras a ampliar el tiempo asignado. Aún no está claro si se extenderá el tiempo o cuánta será la extensión, aunque los parámetros de cualquier aumento probablemente estén entre los dos y los ocho meses adicionales.

\section{Transnacionalización en una facultad de educación de Ontario}

Como ya se mencionó, probablemente se deba considerar la transnacionalización como un proceso que podría ocurrir durante un largo período de tiempo, más que como un hecho específico. Como en el caso de McGill, es difícil determinar el momento de nuestro pasado colectivo en que empezamos a dar los pasos que se podrían considerar como el inicio del camino hacia la transnacionalización de nuestro programa de educación inicial docente. Puede haber sido en los años ochenta cuando empezamos a utilizar "bio datos" (información personal) como parte importante de nuestros procesos de admisión, además de la información académica obtenida de los documentos de postulación. También fue durante esos años que invitamos a Donald Schön a visitar nuestra facultad en dos ocasiones para conversar sobre la práctica reflexiva. O quizá fue en 1995 cuando, bajo la dirección de un nuevo decano y después de muchos años, llevamos a cabo la primera gran revisión del programa. También pudo haber ocurrido a inicios de la década del dos mil, cuando un nuevo decano lideró un proceso para replantear la visión de nuestro programa. En ninguna de aquellas ocasiones hablamos de que se tratara de pasos del programa hacia la transnacionalización aunque, visto en retrospectiva, se podría considerar que todas ellas lo eran.

A continuación, identificaré algunas de las características más destacadas de nuestro programa de formación inicial y las discutiré en función de los temas identificados más arriba. 


\section{Declaración de nuestra vision}

La declaración de la visión de nuestro programa de formación inicial docente es la siguiente:

Nuestra visión del graduado de la Facultad de Educación de la Universidad de Queen's es la de un profesional con capacidad de reflexión crítica. Se espera que los graduados integren conocimientos teóricos, prácticos y experienciales para la comprensión y resolución de problemas a nivel profesional. Vemos al profesor principiante como un agente activo en el desarrollo de una pedagogía inclusiva desde el punto de vista social, orientada a la justicia social. Desde nuestra perspectiva, el docente con capacidad de reflexión crítica es aquel que plantea preguntas que van más allá de los avatares inmediatos de la práctica diaria y que está dispuesto a trabajar de manera colaborativa con otros profesionales de su área y con todos aquellos involucrados con la formación y desarrollo de los estudiantes.

\section{Características del programa}

- El programa está comprometido con la excelencia académica y con el aprender a aprender y además refleja la enseñanza como una actividad tanto intelectual como práctica, de acuerdo con los principios de Queen's.

- El programa presupone que todos los candidatos tengan las habilidades críticas y de lecto-escritura que se asocian con un individuo de su nivel académico.

- El programa promueve la vocación de servicio como un valor fundamental en la profesión del educador y la inclusión como un principio pedagógico básico.

- El programa integra los siguientes dominios: contexto escolar; currículum; enseñanza y aprendizaje; valoración, evaluación y reporte; fines, propósitos y valores educacionales; además de sus bases históricas y filosóficas.

- Las siguientes temáticas se encuentran de manera intrínseca en el programa: inclusión y justicia social; colaboración y liderazgo; el uso de tecnologías de la información y comunicación para la enseñanza y el aprendizaje.

- El programa busca preparar futuros profesionales que aborden temáticas relacionadas con la sostenibilidad en sus aulas, desde una actitud consciente sobre la realidad ambiental, la práctica de la conservación de recursos y la exploración de nuevas maneras de minimizar el impacto del ser humano sobre el medio ambiente. ${ }^{6}$

La declaración de la visión de nuestro programa de entrenamiento para docentes novatos evoca un lenguaje coincidente con las temáticas de la educación transnacional. La idea de un profesor capaz de reflexionar de manera crítica como aquel que se plantea interrogantes más allá de las presiones cotidianas de la práctica diaria, sugiere 
por cierto la temática de sobrepasar las barreras y formatos tradicionales. Elementos como el concepto del profesor como un agente activo en el desarrollo de una pedagogía inclusiva socialmente, orientada a la justicia social, la vocación como elemento central de la profesión docente y la inclusión como un principio pedagógico fundamental, hablan de una disposición del docente a mirar hacia afuera, con apertura de mente, con mayor conciencia acerca de problemáticas transculturales y más aceptación de otras culturas sin prejuicios; igualmente sucede con la voluntad de conocer de manera profunda más que superficial las culturas, las tradiciones y los valores de los estudiantes y con la orientación hacia la justicia social. La idea de que el programa integre la comprensión de los fines, propósitos y valores educacionales al igual que de sus bases filosóficas e históricas también sugiere la predisposición del docente hacia un conocimiento profundo más que superficial, como lo señala el compromiso con la excelencia académica y con el aprender a aprender y la consideración de que todos los candidatos docentes deben tener las habilidades críticas y de lecto-escritura asociadas a su nivel educacional. Las declaraciones de visión acerca de aprender a aprender, de la reflexión crítica, de la colaboración y el liderazgo se relacionan con el enfoque temático del desarrollo profesional contínuo, la práctica reflexiva y el desarrollo de comunidades de aprendizaje.

En resumen, aunque nuestra visión acerca del programa de formación inicial docente en realidad no especifica la concepción de un programa transnacionalizado, muchas de las ideas que expresa coinciden con temáticas comunes de la educación transnacional y con el perfil de los profesores transnacionalizados. Ninguna de sus ideas parece ser contraria a las temáticas y disposiciones de la formación docente transnacional.

\section{Nuestro proceso de admisión}

Nuestro proceso de admisiones utiliza tanto datos académicos como "bio datos" presentes en el proceso. El registro académico es evaluado al examinar las transcripciones previas de manera bastante típica y esto nos ayuda a definir cuáles de los participantes cumplen con características que los predisponen a la excelencia académica. Una declaración escrita personal de los postulantes acerca de su experiencia nos ayuda a determinar quiénes de ellos están predispuestos a la justicia social, la vocación, el liderazgo y la colaboración, la reflexión crítica, al igual que a las habilidades en lectoescritura. En otras palabras, utilizamos la declaración personal para intentar determinar cuáles de los postulantes ya cumplen con una predisposición hacia las cualidades del docente descritas en nuestra declaración de visión. Dado que esta última coincide con los temas y las disposiciones definidas para la educación transnacional (según se indica más arriba), el proceso de admisiones también es coherente con las temáticas de la transnacionalización en educación.

En el caso del programa de posgrado de ocho meses, que en realidad es muy breve como para intentar muchas actividades de formación personal con nuestros estudiantes, damos igual nivel de importancia al registro académico y a la declaración personal. Los postulantes tienen bastantes años más de madurez en relación con 
alumnos recién egresados de la educación secundaria y, en general, su predisposición y características ya se han definido. A su vez, en aquellos estudiantes que llegan al programa directamente de la educación secundaria y que aún están en proceso de definir el tipo de persona que quieren ser, la declaración personal tiene menor influencia final puesto que disponemos de más tiempo para trabajar con ellos durante los cinco años de duración del programa.

En resumen, tenemos un proceso de admisiones que parece ser compatible con las temáticas y disposiciones de la educación docente transnacional.

\section{Nuestros candidatos a docentes}

Como norma general, nuestro proceso de admisiones parece dar como resultado candidatos a docentes que tienden a representar, en distinta medida, algunas de las predisposiciones personales de los profesores transnacionales efectivos. Durante mis treinta y cinco años de experiencia en la facultad, la composición de la clase del programa de formación inicial ha cambiado de manera notable. En el pasado, los estudiantes de la clase eran predominantemente blancos; ahora un porcentaje importante de ellos pertenece a minorías. Muchos de nuestros candidatos a docentes llegan desde Toronto, que se ha convertido en una urbe de inmigrantes de todas partes del planeta, lo cual se refleja en nuestra clase. Muchos de aquellos seleccionados para nuestro programa pertenecen a la primera o segunda generación de inmigrantes, todos los cuales se comunican en inglés fácilmente, mientras muchos de ellos hablan otros idiomas y tienen profunda experiencia personal en otras culturas y sistemas educativos. Por otra parte, varios de los candidatos no inmigrantes han tenido experiencias significativas en el extranjero, mediante experiencias laborales o académicas, o a raíz de vivir en el extranjero con sus padres o familiares, o bien gracias a extensas experiencias de viaje, incluida la de "mochilear." Estos factores podrían sustentar temas como una amplia y profunda conciencia acerca de problemáticas transculturales, aceptación de otras culturas, habilidad de pensar dentro de sistemas culturales y valóricos alternos y la adopción de un enfoque comparativo, aprovechando las diferencias lingüísticas y culturales que pudiese existir en el aula para mejorar el aprendizaje en vez de considerarlas como obstáculos. En resumen, aunque generalmente ignoremos las posibilidades, nuestros candidatos a docentes bien podrían ser uno de nuestros recursos más potentes en la labor de desarrollar un poderoso programa transnacional de educación docente. Aún no hemos reconocido los recursos que tenemos ni hemos aprendido a aprovechar su máximo potencial.

\section{Nuestro enfoque en la práctica reflexiva}

Según se establece en la declaración de nuestra visión, nuestro programa enfatiza la práctica reflexiva. De hecho, hablamos tanto de este tema que, en ocasiones, se ha sabido de estudiantes que indican que están cansados de reflexionar. Además de ejercicios sencillos de reflexión escrita, las dos maneras principales en que incorporamos esta práctica al programa son la tarea de investigación acción y la tarea de portafolio 
profesional que se presenta ante un panel de pares. Todas estas se relacionan con el énfasis de la transnacionalización en el desarrollo profesional continuo, la práctica reflexiva y el desarrollo de comunidades de aprendizaje.

\section{Nuestros cursos}

En cuanto a los contenidos de nuestro currículum y cursos de preparación, nos enfocamos en las directrices curriculares definidas por el Ministerio de Educación de Ontario. Existe poco y nada de esfuerzo por integrar documentos curriculares de otras jurisdicciones para un análisis comparativo; además, para ser honesto, habría poco tiempo disponible para esto debido a lo muy acotado del calendario de nuestro programa (el programa de derecho de McGill obtiene sus logros en cinco años, en comparación con los tres años de los programas de derecho tradicionales en Canadá). Los documentos de Ontario presentan resultados de aprendizaje a lograr y dan libertad al profesor para seleccionar los materiales y las metodologías del aprendizaje que utilizará para llevar a sus estudiantes a cumplir con dichas expectativas. Es así que, esencialmente, en Ontario no hay una verdadera barrera que superar en términos de materiales y metodologías de aprendizaje, en lo que respecta al gobierno jurisdiccional. Como el gobierno no determina los materiales y las metodologías, es nuestro deber enseñar acertadamente los conceptos de planificación y presentación de clases. Este último objetivo al parecer lo logramos con relativo éxito, dado que nuestros graduados luego van a enseñar a otras regiones de Canadá y el extranjero y lo hacen de manera satisfactoria.

Idealmente, la inclusión y la justicia social deberían ser temáticas intrínsecas a lo largo de nuestros cursos de formación inicial lo cual, hasta cierto punto, está ocurriendo en algunos cursos. Tenemos determinados cursos que se relacionan directamente con las temáticas identificadas en la educación docente transnacional; tres de ellos son electivos de "profundización"(FOCI, por su sigla en inglés):

- FOCI 202 Educación global mediante la cooperación internacional

- FOCI 255 Educadores en el extranjero

- FOCI 296 Enseñanza para la justicia social

El contenido de cada uno de los cursos mencionados se relaciona con una o más de las temáticas transnacionales y tiene el potencial para convertirse en una contribución importante al programa transnacionalizado, sin embargo, a excepción de Educadores en el extranjero, casi nunca se completan sus cupos. Tenemos dos módulos breves que abordan los contextos históricos, políticos, filosóficos y sociales de la educación, pero en general estos son mirados con desdén por muchos candidatos que no ven su relevancia debido al breve tiempo que se les asigna y a su aparente desconexión del resto del currículum del programa. Para resumir, aunque contamos con algunos cursos que podrían ser de utilidad, en general a nuestros cursos les vendría muy bien un rediseño profundo, en caso que buscáramos de manera consciente un programa transnacionalizado. 


\section{La práctica}

Nuestros candidatos a docentes de la zona de Toronto habitualmente quieren regresar a su localidad para su práctica profesional, lo cual intentamos hacer en la medida de lo posible. Muchos son enviados a escuelas donde la población de estudiantes y también con frecuencia la de profesores y personal es transnacionalizada. En estos casos el aprendizaje que ocurre durante la práctica parece estar más directamente relacionado con el aspecto de la mirada centrada en lo exterior, con apertura de mente, mayor consciencia de problemáticas interculturales y mayor aceptación de otras culturas.

\section{La práctica alterna}

Como se indicó anteriormente, la práctica alterna tiene por finalidad permitir al candidato acceder a una experiencia educativa seleccionada por sí mismo, la cual le entregará una perspectiva diferente de aquella de un profesor de aula de una escuela pública de Ontario. De esta manera, pueden desempeñarse en una escuela privada de Ontario, en una escuela pública de otra provincia o país y muchas otras opciones. La única limitación son los aspectos de seguridad y la capacidad del candidato para entregar una justificación razonable acerca de cómo y por qué la experiencia propuesta le ayudará a convertirse en un mejor docente; muchos candidatos son creativos e ingeniosos para proponer su experiencia. Cada año, entre 50 y 80 candidatos eligen desarrollar esta experiencia práctica en otros países incluyendo, aunque no de manera única, países tercermundistas. A menudo enseñan en escuelas de elite donde el lenguaje de enseñanza es el inglés, aunque en los últimos años un número pequeño pero creciente de candidatos ha seleccionado escuelas en contexto indígena de África y El Caribe. Algunos han elegido escuelas en zonas urbanas pobres de Inglaterra. Algunas selecciones típicas también incluyen el Sur y el Este de Asia, Sudamérica, Europa y Oriente Medio; algunos pocos eligen el extremo Norte de Canadá. La facultad cuenta con recursos destinados a financiar los viajes de los candidatos a ubicaciones en el extranjero y, de cumplirse ciertas condiciones, los candidatos pueden postular para obtener financiamiento. Generalmente alrededor de unos 60 candidatos llegan a recibir financiamiento parcial para cubrir los costos de sus viajes; otros que seleccionan proyectos en desarrollo hacen actividades para reunir recursos, mientras que otros financian sus gastos de manera particular. Varios de estos candidatos describen su experiencia de práctica alterna como una que "les cambió la vida." Nuevamente, el aprendizaje que ocurre durante estas prácticas alternas parece estar relacionado de manera más directa con el aspecto de la mirada centrada en lo exterior, con apertura de mente, mayor consciencia de problemáticas interculturales y mayor aceptación de otras culturas, al igual que con el tema de la orientación hacia problemáticas de justicia social. 


\section{La Feria de Reclutamiento para Profesores en el Extranjero (TORF, por su sigla en inglés)}

Cada mes de febrero desde hace más de 25 años, personal de nuestra Oficina de Servicios para la Carrera de Educación, ha organizado la Feria de Reclutamiento para Profesores en el Extranjero (TORF, por su sigla en inglés), considerada la tercera más importante en toda Norteamérica. Este evento congrega a más de 90 reclutadores de escuelas internacionales de todo el mundo y a alrededor de 300 docentes que esperan conseguir una oportunidad laboral en el extranjero. La mayoría de los candidatos son experimentados docentes, pero una fracción de los puestos está reservada para miembros postulantes al grado de nuestro programa. Aunque la Feria no es considerada parte formal de nuestro programa, estimamos que esta experiencia hará del graduado que haya enseñado en otro país un mejor profesor una vez que regrese a Canadá y a Ontario. Muchos de nuestros graduados que obtienen su primera oportunidad laboral gracias a la Feria, regresan a Canadá luego de un contrato de dos años, sin embargo algunos deciden hacer su carrera enseñando en el extranjero. Sea cual sea el resultado, este será congruente con los temas de la educación transnacional de docentes, en particular con una mayor consciencia de problemáticas interculturales y mayor aceptación de otras culturas. Un beneficio colateral de organizar la Feria ha sido que el personal de la Oficina de Servicios para la Carrera de Educación ha desarrollado un red de contactos confiables en escuelas internacionales y pueden asesorar a los candidatos en la búsqueda de oportunidades para su práctica alterna.

\section{Factores que favorecen la transnacionalización}

Nuestro programa de formación inicial docente cuenta con una serie de cualidades que conforman una base sólida para el desarrollo a futuro de un programa transnacionalizado. Tanto la declaración de la visión, nuestro proceso de admisiones y el perfil de candidatos a docentes que arroja, nuestro énfasis en la práctica reflexiva como el componente de la práctica alterna son factores de solidez que favorecen acciones futuras hacia una mayor transnacionalización del programa. La práctica profesional y la Feria de Reclutamiento para Profesores en el Extranjero pueden ser considerados como factores de apoyo en este punto. Describo a la práctica como apoyo más que como factor decisivo sólo debido a que no todos los candidatos pueden conseguir un puesto en una escuela o en una sala transnacionalizada.

Nuestra ubicación geográfica, no tan lejana a Toronto, también es un factor positivo. A los canadienses nos gusta vernos como una nación multicultural; sin embargo, de acuerdo con wiki.answers.com, "la multiculturalidad de Canadá se encuentra principalmente en sus ciudades más importantes: Toronto, Montreal y Vancouver. Por ejemplo, apenas sobre el 50\% de los habitantes de Toronto no nació en Canadá. Sin embargo, aparte de un par de ciudades principales, Canadá es un país de población más bien caucásica." 7 Nuestra capacidad de atraer a candidatos de Toronto y otorgarles oportunidades de práctica profesional allí es definitivamente un atributo 
en términos de transnacionalización. Claramente, los centros de menor densidad también tienen poblaciones de inmigrantes y muchos de nuestros graduados llegarán a desempeñarse en ellos.

En cuanto a los factores jurisdiccionales, el hecho de que, en tanto cumplamos con los requerimientos básicos sobre currículum y marco de tiempo establecidos por las regulaciones de Ontario, tengamos libertad para ir más allá de estos es un factor positivo. Nuestra visión implica nuestra voluntad de ir más allá de las presiones inmediatas de la práctica cotidiana. Es de esperar que el gobierno decida extender el programa, lo que contaría como un factor positivo adicional ya que proporcionaría tiempo para dedicar a una mayor integración de los contextos filosóficos, políticos, sociales e históricos al programa y a una mayor cantidad de actividades de aprendizaje que ayuden a relacionar los valores subyacentes y las tradiciones intelectuales con las prácticas educativas actuales.

\section{Factores que dificultan la transnacionalización}

Además de los problemas organizacionales que implica motivar a los miembros de una facultad para la toma de decisiones y para participar en acciones para orientadas a implementar cambios en los programas, al igual que cualquier obstáculo relacionado con escases de recursos económicos, identifico cuatro factores que dificultan una eventual transnacionalización de nuestro programa de formación inicial docente. Estos factores son: las limitaciones de tiempo del programa de ocho meses; nuestra falencia en la labor de aprovechar nuestro equipo transnacionalizado de candidatos a docentes como un poderoso recurso para la enseñanza y el aprendizaje; la problemática sobre cómo ayudar a los candidatos a relacionar con claridad los valores subyacentes y las tradiciones filosóficas con las prácticas educativas actuales; y la problemática acerca de cómo integrar la investigación comparativa en cada uno de nuestros cursos para promover una comprensión más profunda de diversas interrogantes. Puede ser necesario "reasignar" a algunos miembros de la facultad.

\section{Conclusiones}

Debo admitir que al finalizar este estudio mi impresión acerca de la factibilidad de la transnacionalización de los programas de formación inicial de docentes es mucho más positiva de lo que era al inicio. Para ser honesto, me sorprendió bastante darme cuenta de cuánto coincide nuestra declaración de visión con los principales temas de la transnacionalización en la formación docente, además del hecho de que mi propia facultad ya cuenta con una base sólida sobre la cual construir un programa más transnacionalizado. Fue emocionante darme cuenta de que tenemos un recurso muy significativo que nos podría ayudar a transformar nuestro programa, pero que prácticamente lo hemos ignorado. También me sorprendió notar que casi no hay verdaderas restricciones jurisdiccionales más allá de la brevedad del tiempo que el gobierno de Ontario respalda financieramente el programa. Con algo de reflexión, planificación, esfuerzo y compromiso, podríamos transformar nuestro programa en 
una versión mucho más transnacionalizada y así obtener beneficios para nuestros candidatos a docentes y para sus futuros estudiantes.

Finalmente, el punto central es: He utilizado a mi propia Facultad como caso de estudio. Aunque nuestro programa estaba a la vanguardia cuando introdujimos cambios significativos en los años noventa, muchas otras facultades de Ontario han llegado a nuestro nivel desde entonces. De esta manera, si contamos con una base a partir de la cual transformarnos, es probable que otras facultades de Ontario también la tengan, aunque su base sea diferente a la nuestra. En resumen, mi conclusión es que la transnacionalización puede ser un paradigma viable para la formación inicial de docentes en Ontario.

\section{Notas}

1. Facultad de Educación, Queen's University, Kingston, Ontario, Canadá. Decano asociado interino 1994-1996 y Decano asociado 1996-2008.

2. Mi colega de la Queen's University, Tom Russell, podría describir mis reflexiones como escritos desde "la autoridad que entrega la experiencia." Ver, por ejemplo, la discusión entre Munby y Russell (1994) sobre la autoridad de la experiencia en el aprendizaje para enseñar física en el nivel secundario. Su trabajo se basa en los escritos de Schön $(1983,1987)$. Para un punto de vista algo más escéptico, ver, por ejemplo, la discusión de Mack y Delicio (2000) acerca de la tensión entre el conocimiento profesional y la experiencia individual en sus evaluaciones acerca del uso de las tecnologías de la información en el aula. En la Queen's University, la práctica reflexiva es un elemento y componente clave del programa de formación inicial de docentes y el acuerdo colectivo entre la Universidad y la Asociación de Profesores de la Facultad reconoce de manera específica el autoaprendizaje de los docentes como una forma legítima de actividad académica para los instructores de todas las facultades y departamentos.

3. El cambio de marca de instituciones y programas bajo la dirección de consultores en marketing de ese ámbito es una tendencia actual entre las escuelas profesionales y universidades canadienses y hay demasiadas experiencias de ese tipo. Ese no es el tipo de transformación que deberíamos buscar. Por otra parte, puede ser necesario recurrir a la ayuda profesional para promocionar nuestros programas transformados de manera genuina, con el fin de atraer a un tipo de postulantes que no sólo desee integrarse a los programas que ofreceremos, sino que también cuente con las características personales que contribuirán a su éxito tanto en los programas como en su desempeño profesional.

4. Me atrajo el concepto de "zonas fronterizas" en el título del artículo. Aunque la zona fronteriza a la que se refiere es un lugar real, el concepto es simbólico y, en mi opinión, se puede considerar más amplio que el concepto de transnacionalismo. Aunque desde luego incluye a los hijos de inmigrantes y a los hijos inmigrantes y migrantes, también puede incluir a otros que vivan en fronteras metafóricas, como pueblos originarios, niños Métis e Inuit en Canadá o nativos americanos en Estados Unidos, e incluso niños con necesidades especiales.

5. Algunas facultades de Ontario, incluyendo a la nuestra, también ofrecen un programa "concurrente" en el cual los candidatos trabajan para cumplir con los requisitos del grado inicial y con los del grado de formación docente de manera simultánea. Por lo general, el tiempo total requerido es casi equivalente (cinco años, o diez semestres). 
6. Esta declaración fue escrita por Dr. Rosa Bruno-Jofré, decana entre 2000-2010 y aprobada por varios comités y la asamblea de la facultad "Faculty Board." Note que la decana es historiadora de la educación, originaria de Argentina y tiene una experiencia internacional muy extensa y ha estado expuesta a desarrollos en varios contextos culturales y políticos. Universidad de Queen's, Calendario 2012-2013 de la Facultad de Educación. Disponible en línea en: http://www.queensu.ca/calendars/education/.

7. http://wiki.answers.com/Q/Canada_is_very_multicultural_so_what_is_ the_most_popular_race_in_Canada_and_if_you_had_time_the_second_and_the_ third\#ixzz1xweTB7Po

\section{Referencias}

Cline, Z. and Necochea, J. (2006). Teacher dispositions for effective education in the borderlands. The Educational Forum 70(3), pp. 268-282. [On-line] Disponible: http:// dx.doi.org/10.1080/00131720608984902

Jukier, R. (June 2006). Transnationalizing the legal curriculum: How to teach what we live. Journal of Legal Education 36(2), pp. 172-189. [On-line] Disponible: http://ssrn.com/ abstract $=2002272$ 